\title{
Microwave microscopy applied to EMC problem: Visualisation of electromagnetic field in the vicinity of electronic circuit and effect of nanomaterial coating
}

\author{
Jérôme Rossignol ${ }^{1}$, Didier Stuerga ${ }^{1}$, Guillaume Bailly ${ }^{1}$, Amal Harrabi $^{1}$, \\ Sébastien Girard ${ }^{2}$, and Sébastien Lalléchère ${ }^{2, *}$ \\ ${ }^{1}$ Laboratoire Interdisciplinaire Carnot Bourgogne, Département Interface, GERM, \\ CNRS UMR 6303, Université Bourgogne Franche-Comté, Dijon, France \\ ${ }^{2}$ Université Clermont Auvergne, CNRS, SIGMA Clermont, \\ Institut Pascal, 4 av Blaise Pascal, 63000 Clermont-Fd, France \\ *corresponding author, E-mail: sebastien. lallechere@uca.fr
}

\begin{abstract}
This proposal is devoted to a collaborative approach dealing with microwave microscopy experiments. The application is dedicated to an electromagnetic field cartography above circuits and the influence of nanometric material layer deposition on the circuits. The first application is associated to a microstrip ring resonator. The results match with the simulated fields. The second application is focused on the effects of a dielectric layer deposited on the circuit and its impact in terms of electromagnetic propagation.
\end{abstract}

\section{Physical context and problem statement}

Introduction of nanometric element will be one of the most important challenge of this century in electronics and ElectroMagnetic (EM) applications. Recent functionalities in microelectronic have induced an increasing development of new materials within integrated circuit (IC) and particular systems to characterize them $[1,2]$. The radiated emission or immunity of a device are estimated either in time domain [3] or frequency domain. The present work is focused on the frequency approach. To identify interferences within the circuit, it is possible to combine simulation and experimental approaches [4]. The existing simulation tools include several commercial solutions (HFSS $囚$, CST $囚 \ldots$...) with different numerical methods such as Finite Element Method (FEM), Finite Difference in Time Domain (FDTD) for instance. On the one hand, the main advantage of these tools relies on their capability to handle more and more complex cases, in widespread frequency domains [5], and various applications [6]. On the other hand, their main drawback is often the difficult trade-off between computing costs (time, memory) and providing an accurate picture of the reality. It is all the more the case when dealing with multi-scale material such as ones characterised by different crystalline phases and/or micro- / nano-structures (e.g. works in [7] where antennas' design combined measured and simulated data with magnetic materials).

Obviously, the most common way to perform EM testing (and especially for Electromagnetic Compatibility,
EMC, issue) is to achieve measurements. Assuming a prototype of the equipment under test is available and without exhaustiveness, different experimental devices may reveal useful for EMC compliance tests [8]. Some of them are based upon the generation of plane wave systems such as Anechoic Chambers (ACs) i.e. Faraday's cages embedded with absorbing materials on walls, striplines, Transverse ElectroMagnetic (TEM) cells, and Gigahertz Transverse ElectroMagnetic (GTEM) cells. Whether previous devices are useful to complement theoretical developments for immunity and/or emission EMC testing, they are mandatory for industrial and standard procedures. In this context, several tests systems are conceivable alternatives; we may think about Mode Stirred Reverberation Chambers (MSRCs) based upon the physical pinciples of ideal resonant cavities [9]. Similarly to ACs, MSRCs are made of metallic walls (i.e. Faradays' cages), whereas mode stirring may be obtained from a mechanical stirrer or frequency source stirring. Depending on the sizes of the cage, an inner Working Volume (WV) is predefined where the electromagnetic fields (mostly electric part) is assumed statistically isotropic and homogeneous over an entire rotation of the stirrer (respectively regarding frequency stirring). The main aim regarding mechanical frequency stirring is to offer a variety of conditions (boundary and source ones) to generate a diversity of EM sources. This quality of the test procedure is assessed by mean of statistical EM fields' criteria. Both immunity and radiated tests may be achieved in MSRCs, in particular emission of PCB systems and susceptibility to external environment.

In this proposal, the experimental approach is based on local measurements close to the electronic device $[3,10$, 11] (Near-Field, NF, scanning). The handheld measurement is a debug-method to find quickly the root-cause of the EMC problem. Measurements can be fine-tuned and performed easily on different spots. NF cartography is based on an automatic local mapping of measurement. Contrary to the classical method, the reproducibility of the position is assumed. However, this device is expensive and not suitable for multiscale measuring. 
Only few studies about the ElectroMagnetic Interferences (EMIs) have combined material science and (EMC) [12], even if the importance of material science in EMC is known for a long time [13]. The authors develop a collaborative project which associates these disciplines, using the microwave microscopy [14] along with numerical simulation comparisons. The objectives are to explore the influence of new materials (such as nanometric layers) on circuits and their effects on the distribution of EM fields.

\section{Experimental and numerical test benches}

\subsection{Experimental setup}

As shown in Fig. 1, the experimental set-up is composed of a positioning platform including XY- and Z-components with respectively spatial resolutions of $10 \mu \mathrm{m}$ and $5 \mathrm{~nm}$ at horizontal (XY) and vertical (Z) levels. The device under test is placed on the XYZ stage in order to move the circuit under the electric probe (fixed with its own cable). Thus, the analysis system excludes all mechanical stress on the probe which would induce significant perturbations during the measurements. It is composed of home-made E- and H-probes $[15,16]$ connected to a spectrum analyzer which may be replaced by a Vector Network Analyzer (VNA). The whole setup is monitored by LabVIEW(C) software through General Purpose Interface Bus (GPIB). Using a laser profilometry, the surface-to-probe distance is controlled and defined as a constant parameter.

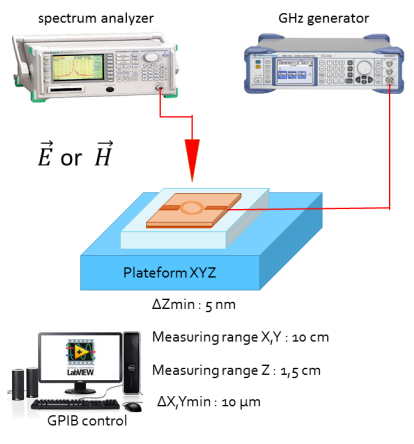

Figure 1: Experimental set-up including spectrum analyzer, $\mathrm{GHz}$ generator and XYZ-platform.

\subsection{Process}

Experimental results are obtained via a sequence of operations consisting in several steps (Fig. 2). First, laser mapping of the surface allows fixing the probe-to-sample distance; thus EM scanning is performed with respect to this distance. Then, instruments are settled into an embedded micro-anechoic chamber. Measuring has been achieved over an area of $33.5 \times 33.5 \mathrm{~mm}$ : the number of points is $68 \times 68$ (focusing on the ring), and step size is fixed to $0.5 \mathrm{~mm}$. The surface-to-probe distance is chosen to be wide: $6 \mathrm{~mm}$ as shown in figure 2 . The spectrum analyzer measures, at each position $\mathrm{X}[\mathrm{i}]$ / $\mathrm{Y}[\mathrm{j}]$, the magnitude of electromagnetic radiation caused by the sample, detected by the $\mathrm{E} / \mathrm{H}$ probe versus frequency within the entire band range of the instrument ( $\mathrm{i}$ and $\mathrm{j}$ are associated respectively to the indices of $\mathrm{X}$ and $\mathrm{Y}$ coordinates). Finally, at chosen frequency, postprocessing induces a file composed of the $\mathrm{i} / \mathrm{j}$ locations and the amplitude of radiated levels. Using "ParaView", an open-source multi-platforms data analysis (postprocessing) and visualisation application, a qualitative and quantitative cartography of the radiated emission close to the circuit is achieved.

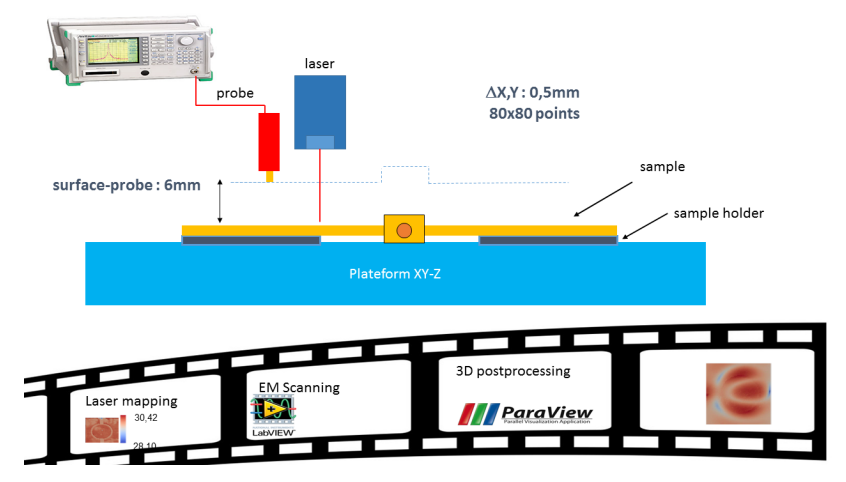

Figure 2: Experimental sequence of the microwave imager.

\subsection{Numerical setup and first $\mathbf{S}$-parameters results}

The numerical configuration is illustrated in figures 3 and 4; the sample is a classical microstrip ring resonator with a resonant frequency at $2.66 \mathrm{GHz}$. It consists in one conducting (copper) ring, two input and output ports (respectively 1 and 2 in Fig. 4), one copper ground plane and FR4-substrate whose thickness and relative permittivity are respectively $h=1.6 \mathrm{~mm}$ and 4.6 .

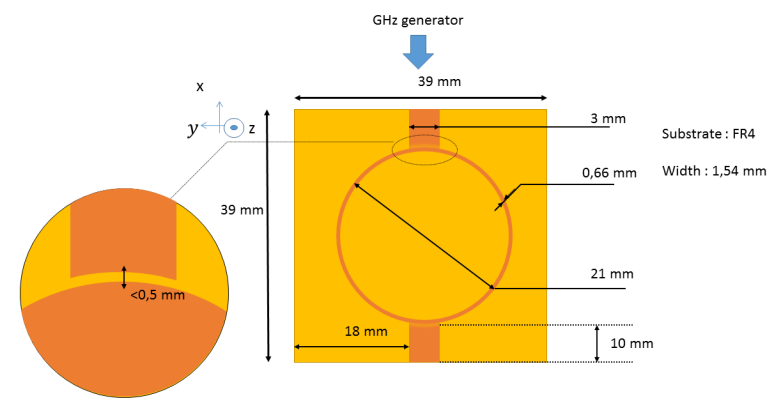

Figure 3: Design of the microstrip ring resonator.

In this work, time domain solver from CSTß) (relying on Finite Integral Technique, FIT, method) is used. The simulation time is set to $100 \mathrm{~ns}$ in order to ensure an energy dissipation inside the Computational Domain (CD) greater than $120 \mathrm{~dB}$ relatively to the maximum of inserted energy. The Cartesian CD is composed of a $74 \times 74 \times 40 \mathrm{~mm}^{3}$ parallelepiped with absorbing boundary conditions. The EM time source is a sine Gaussian pattern applied to port num- 


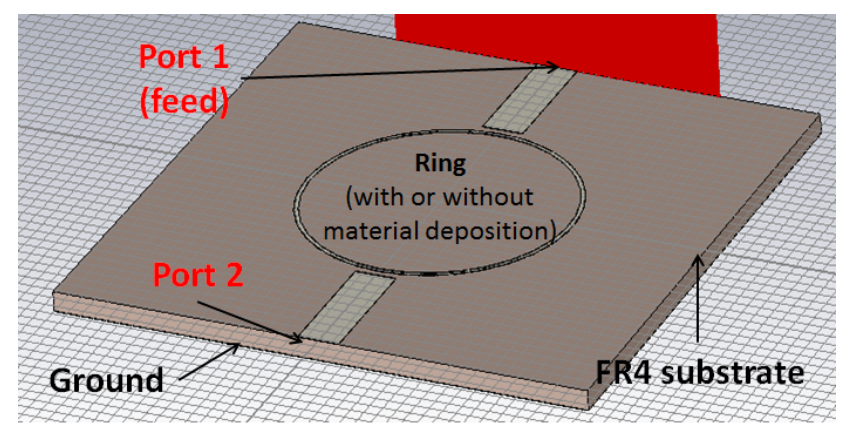

Figure 4: Mockup of the numerical setup following details given in Fig. 3.

ber 1 (waveguide port, see figure 4). In the following, we will lay emphasis on the combination of conducted / radiated results to qualify the proposed procedure both considering numerical and experimental data. Regarding the radiated EM fields, it is to be noted that the observed cartography is located in an area $\left(\approx 34 \times 34 \mathrm{~mm}^{2}\right.$ in XY-plane $)$ at $6 \mathrm{~mm}$ above resonator.

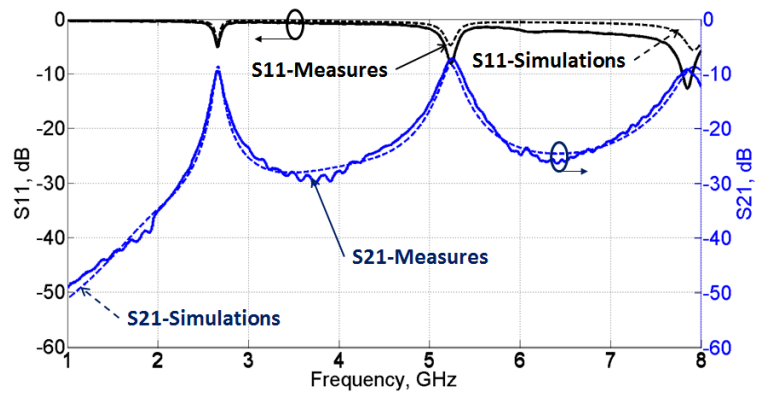

Figure 5: S-parameters from measurements (plain lines) and simulations (dotted lines) in "empty" test case from $1 \mathrm{GHz}$ to $8 \mathrm{GHz}$.

Figure 5 shows the variations of S-parameters both regarding data from measurements and simulations. The responses of the first three resonant modes is shown in these figures. As expected, from a conduction point of view, CST $®$ simulations are very close to the VNA measurements at the resonance frequency; an excellent agreement is also observed from $1 \mathrm{GHz}$ to $8 \mathrm{GHz}$.

As illustrated in figures 3 and 4 , the presence of the material deposit $\left(\mathrm{TiO}_{2}\right)$ is inner to the ring. In the following part, we will lay emphasis on the EM fields radiated from the ring resonator at a given distance of the substrate. It is assumed that the excitation generator is operating at $2.66 \mathrm{GHz}$. EM propagation is, theoretically, mainly defined by Transverse Magnetic (TM) mode [17]. In these conditions, the electromagnetic field is mainly composed of an electric field and a normal magnetic field. Thus, Cartesian $E_{z}$ contribution is the main component of the electric field whereas the magnetic field is defined here by $H_{x}$ and $H_{y}$; in this proposal, only these components are studied.

\section{Electromagnetic impact of material deposition}

This section is devoted both to the simulated and measured results obtained for EM conduction / emission testing.

\subsection{Nanomaterial $\mathrm{TiO}_{2}$}

The assessment of the nanomaterial effect involves here a classical titanium dioxide [18] $\mathrm{TiO}_{2}$. The name of this material is P25 from Degussa (see figure 6 for nanometric view). This raw material is already used in photocatalysis, waste-water treatment, and also in photovoltaic cells. This type of material is present in electronic device inside varistors and MOSFET, or in nano-structured form in Libased batteries (e.g. aerospace application); its deposition as a protective coating (for instance against corrosion) may be achieved near the electronic system. It is also to be noticed this material is used as a coloring pigment. From a chemical point of view, this material is composed of two cristallographic phases of $\mathrm{TiO}_{2}$ anatase and rutile $[19,20]$.

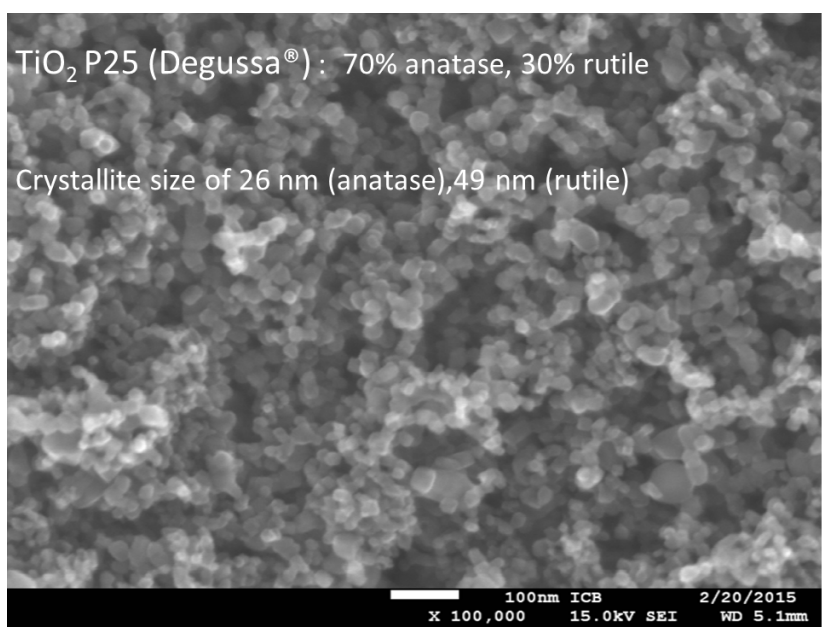

Figure 6: Scanning Electron Microscopy (SEM) view of $\mathrm{TiO}_{2}$ P25 nanomaterial. The morphology and size of elements at nanometric scales is to be noticed here.

It is to be noticed that the resonant frequency changes depend on the morphology of $\mathrm{TiO}_{2}$ material and of coating. The following subsections will illustrate the effect of $\mathrm{TiO}_{2}$ both for conduction (i.e. S-parameters relatively to previous section, see subsection 3.2) and radiated EM fields (see subsections 3.3 and 3.4).

\subsection{S-parameters in presence of $\mathrm{TiO}_{2}$ and methodol- ogy}

As aforementioned, the aim of this section is to put the focus on the impact of the material inner deposition at ring location. Figure 7 stands for the S-parameters variations of the system from $1 \mathrm{GHz}$ to $8 \mathrm{GHz}$ with inner material (see Fig. 4).

Figure 7 focuses on the variations of S-parameters (i.e. S11 and S21) given by simulations and measurements. It 


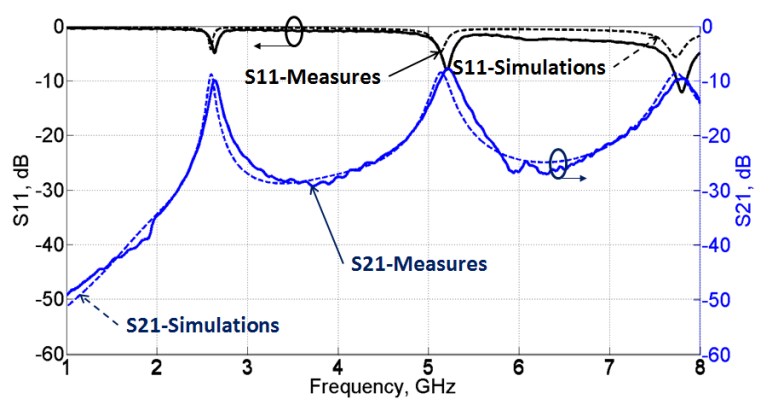

Figure 7: S-parameters from measurements (plain lines) and simulations (dotted lines) in presence of $\mathrm{TiO}_{2}$ material from $1 \mathrm{GHz}$ to $8 \mathrm{GHz}$.

is worth-noticing that the material $\left(\right.$ here $\left.\mathrm{TiO}_{2}\right)$ layer is obtained according to a simple "Doctor Blade" protocol with a thickness estimated around $36 \mu \mathrm{m}$ (laser profilometer). Obviously, figure 7 shows the EM impact of $\mathrm{TiO}_{2}$ deposit in comparison with "empty" case (Fig. 5). As expected, the presence of material, whose relative permittivity is assessed around 6.4 (value obtained in previous works [21, 22]), involves slight decrease of resonance frequencies. The good agreement observed between simulations (dotted lines) and measurements (plain lines) validates the model at conduction level (i.e. regarding S-parameters). It is to be noted that the assumption linked to the relative permittivity of $\mathrm{TiO}_{2}$ is experimentally verified by considering frequency shift due to material deposit (see comparisons between measurements with or without $\mathrm{TiO}_{2}$ in figures 5 and 7).

The following subsections will illustrate the effect of $\mathrm{TiO}_{2}$ both for conduction (i.e. S-parameters relatively to previous section, see subsection 3.2) and radiated EM fields (see subsections 3.3 and 3.4).

The main goal of this work is to propose a NF microscopy cartography of a classical electronic system (ring resonator) in its close neighbourhood (i.e. distance $h$ from system to probe: $h \approx l / 7$, with $l$ the characteristic size of the system; here $h=6 \mathrm{~mm}$ and $l=39 \mathrm{~mm}$ ). The first step of this work is to assess the level of the radiated EM fields at height $h$. In this framework and in the following subsections, figures 8 to 12 bring some interesting details about the validity of the proposed approach. Since the major interest in this work has relied on quick qualitative methodology to characterize different electronic systems in EMC framework, it has been decided to propose results relatively to the maximum of EM field respectively obtained from simulations / measurements in "empty" test case (see next part 3.3 for additional details). This means that simulated and measured results are normalized from the maximum observed in each case in figures 8 to 10 . The same principle is followed in subsection 3.4 for simulations and measurements. Finally, in order to keep some comparable data with subsection 3.3 , the normalization procedure is based upon maximum of fields observed in "empty" test case to produce figures 11 to 13 , and is discussed in next parts.

\subsection{Radiated results in "empty" test case}

First of all, results are compared after normalization from numerical/ experimental value. Then, we lay emphasis on the EM fields radiated by the electronic circuit at frequency $f=2.66 \mathrm{GHz}$ and height $h=6 \mathrm{~mm}$ above the ring resonator without material $\left(\right.$ here $\mathrm{TiO}_{2}$ ) deposition. The results are presented in figures 8 to 12 , respectively corresponding to the expression of simulated and measured data for components $\mathrm{E}$ (electric field, mostly normal field), $H_{y}$, and $H_{x}$ (Cartesian magnetic fields). Indeed, as previously theoretically assumed, the propagation is mostly due to TM mode and some results are expected according to work in [17]. Indeed, the results provided in figures 8-10 are in accordance with theoretical expectations. Indeed, both experimental (right) and numerical (left) data in figures 8-10 show the classical distribution of EM fields at resonance frequency $f=2.66 \mathrm{GHz}$.
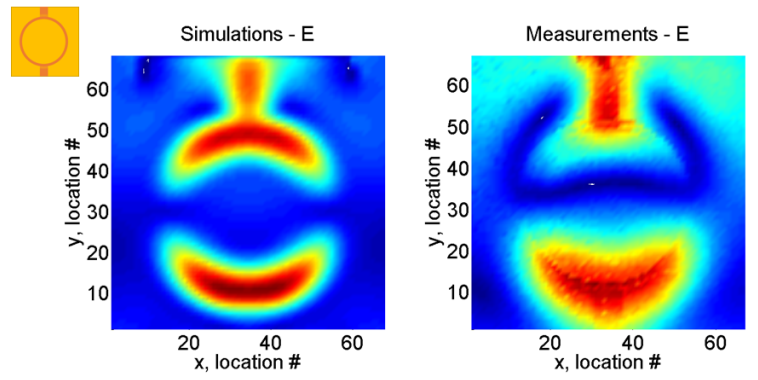

Figure 8: $\left|E_{z}\right|$ magnitude of the E-field for simulations $(\mathrm{CST} \AA$, left) and measurements (right) in the vicinity of the empty ring resonator at $f=2.66 \mathrm{GHz}$.

As shown in figure 8, picture on the left side is a simulated mapping of $\left|E_{z}\right|$ magnitude at a distance surface probe: $6 \mathrm{~mm}$. The right part of the figure stands for the experimental cartography at same distance.

Figure 8 shows similar distributions of E-field regarding simulations (left) and experiments (right). It is to be noticed that the chosen colormap is spread from "relative" minimum (deep blue) to maximum (red) data. The previous remark is also valid for next figures 9 to 13 . However, CST®simulations allow accessing the value of component with a finer sampling comparatively to measurements (without complementary simulations costs). The experiments give an integral value of local area in the vicinity of the circuit.

The resonance behaviour for transverse magnetic field component $\mathrm{Hy}$ is clearly visible in figure 9: both numerical (left) and experimental (right) data show the four lobes due to resonance. The differences observed near GHz-generator (top of the figure) suggest that some coupling may exist with extra-Hy components of EM fields. Four lobes and the supply port are observed. In figure 9 , a comparison between measured and simulated $\left|H_{y}\right|$ shows a good consistence. A symmetrical radiated field is observed in simulations along 

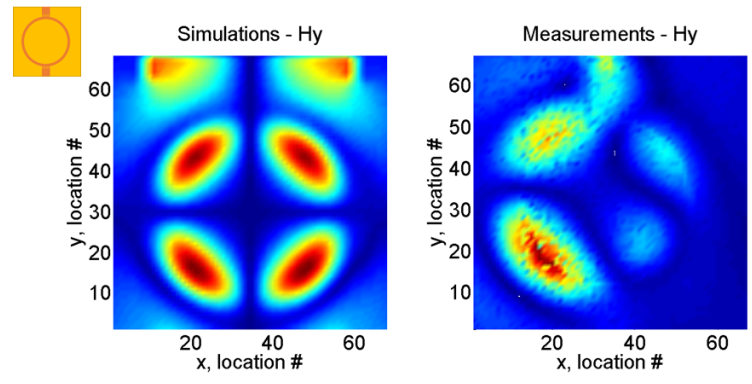

Figure 9: $\left|H_{y}\right|$ magnitude of the magnetic field for simulations (CST $®$, left) and measurements (right) in the vicinity of the empty ring resonator at $f=2.66 \mathrm{GHz}$.

the direction of the excitation port. However, the difference of magnitude between the lobes could be explained by a slight deviation of the probe orientation. It should be noted that the accuracy of the proposed method is also affected by the orientation of the probe, and it is possible to evaluate the three components of the EM field under this condition.
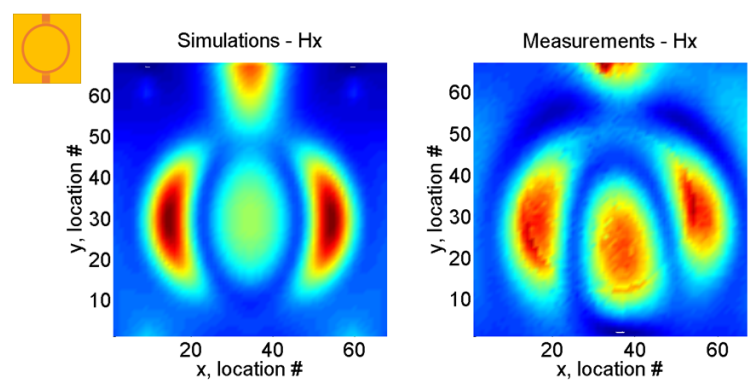

Figure 10: $\left|H_{x}\right|$ magnitude of the magnetic field for simulations (CST $®$, left) and measurements (right) in the vicinity of the empty ring resonator at $f=2.66 \mathrm{GHz}$.

Finally, figure 10 shows component $\left|H_{x}\right|$ which is the magnetic vector normal to the direction of excitation line. Similarly to figure 8, figure 10 lays emphasis on the modal behaviour of the ring resonator at resonance frequency $f$ for magnetic field component $H_{x}$. The "relative" comparison between numerical and experimental results are also in good agreement.

\subsection{Influence of $\mathrm{TiO}_{2}$ material for radiated $\mathrm{EM}$ fields}

This part is devoted to the EM radiations of the ring circuit at frequency $f=2.66 \mathrm{GHz}$ with $\mathrm{TiO}_{2}$ deposit. As expected (and as demonstrated from results in figures 5 and 7), the presence of $\mathrm{TiO}_{2}$ material obviously involves shift of resonance frequencies. The comparison between figures 5 and 7 clearly shows that the assessment of material EM properties (i.e. relative dielectric permittivity) is allowed from Sparameters numerical and experimental measurements; it is in accordance with expected value around 6.4. In this subsection, our interest relies on the evaluation of radiated EM fields, facing data given from previous part (subsection 3.3).
The layer is obtained according to a simple doctor blade protocol with a thickness estimated around $36 \mu \mathrm{m}$ (laser profilometer). Here we present the difference between the simulation and experimental measures.
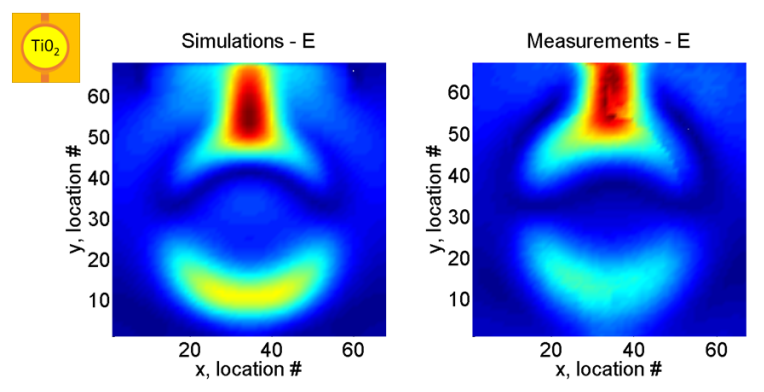

Figure 11: $\left|E_{z}\right|$ magnitude of the E-field for simulations (CST $B$, left) and measurements (right) in the vicinity of the ring resonator covered by $\mathrm{TiO}_{2}$ at $f=2.66 \mathrm{GHz}$.

Results in figure 11 validate the relevance of numerical / experimental tests provided in presence of $\mathrm{TiO}_{2}$ material inside the ring resonator (see figure 4). It is to be noticed that $\mathrm{TiO}_{2}$ deposit leads to a global decrease of the levels of Efield measured above the ring resonator, which is as clearly located as it was the case in figure 8 without material deposition. Previous remark should be tempered since the radiated E-fields above $\mathrm{GHz}$ generator (top of the figure) are strengthened. In a sense, this last observation was expected due to variations around S-parameters and frequency shift (resonance). Indeed, the width of the $\mathrm{TiO}_{2}$ layer induces variations of S-parameters in comparison to "empty" test case. It is assumed a low perturbation of the mode. In the case of the $E_{z}$ component (figure 11), the shape of the experimental signal is similar to CST $囚$ simulations. The difference can be explained by the wide value of the surface probe distance or a slight deviation of probe orientation.
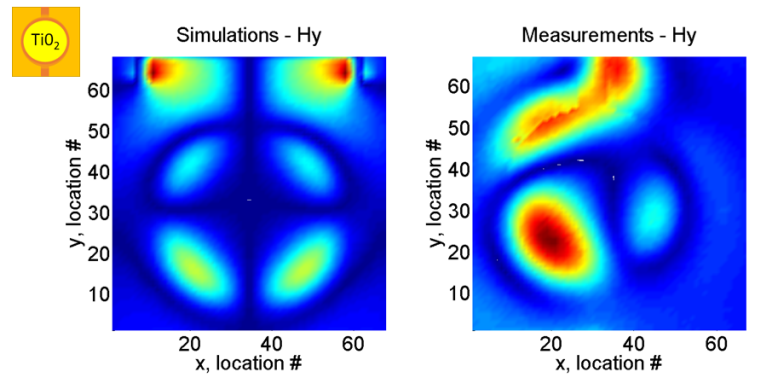

Figure 12: $\left|H_{y}\right|$ magnitude of the magnetic field for simulations (CST $\AA$, left) and measurements (right) in the vicinity of the ring resonator covered by $\mathrm{TiO}_{2}$ at $f=2.66 \mathrm{GHz}$.

The "relative" distribution of Hy-component of EM field show stronger differences between simulations and experiments (e.g. one of the previous four-lobes is missing here, figure 12). Despite all, similarities are observed between numerical and experimental tests: for instance, it is to 
be noted that the two upper lobes (from initial four lobes in "empty" case) are characterized by lower Hy-levels (leading to one lob vanishing in measurements). One of the most likely causes may also be due to the high EM concentration into the layer. The permittivity of the layer is greater than the value associated to the FR4 substrate. In this context, the number of points may reveal insufficient. This cause takes into account the previous observations of the empty ring resonator which is magnified by the high electromagnetic concentration into the layer. The difference can be then explained by the wide value of the surface probe distance or a slight deviation of probe orientation. Therefore, it will be possible to evaluate the three components of the electromagnetic field with nanometric coating. EM concentration within this type of layer induces a sufficient numbers of points and a strict orientation of probe. Despite all, the results obtained are promising since it allows complementary experiments to accurately qualify effect of synthetized circuits with nanomaterials in EMC context.
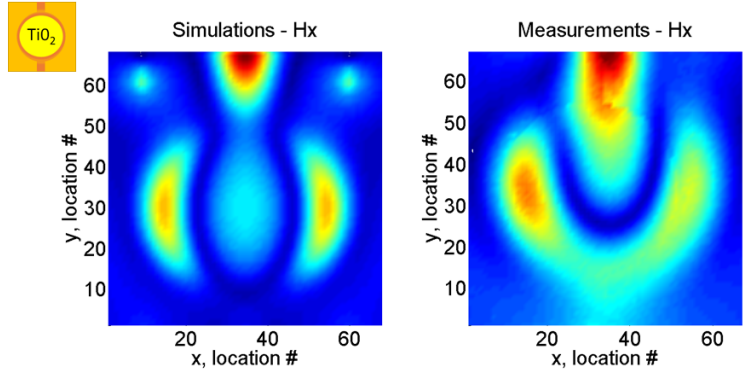

Figure 13: $\left|H_{x}\right|$ magnitude of the magnetic field for simula-

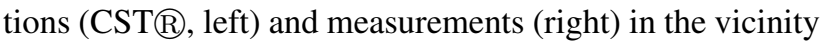
of the ring resonator covered by $\mathrm{TiO}_{2}$ at $f=2.66 \mathrm{GHz}$.

As it was the case in the previous section (3.3), same remarks may be provided from the observation of $H_{x^{-}}$ component both regarding resonance behavioral distribution of fields and clear ring location from EM microscopy measurements (figure 13). Most of EM energy (stored in $\mathrm{CD}$ at frequency $f$ ) is lower than in "empty case" (out of the area surrounding the source generator).

As shown in figure 13, the experimental distribution of the $H_{x}$ magnitude is close to the simulation. A difference between the simulation and the experimental measurement can be also explained by inhomegeneity within the $\mathrm{TiO}_{2}$ layer or an insufficient number of points, or even a slight variation of the probe orientation. "Doctor Blade" coating of the nanometric $\mathrm{TiO}_{2}$, laser profilometry, close evolution of S-parameters between simulation, and experimental measurements dismiss a possible inhomogeneity of layer.

\section{Conclusion}

A combined approach of microwave microscopy experiments and simulation has been presented. A frequency measurement system is proposed for the measurement of the surface EM field of planar circuit. Dedicated to an elec- tromagnetic field cartography above circuits, the first application is associated to a classical microstrip ring resonator ("empty" test case). The experimental results are in a very good agreement with the simulated fields. The second application is focused on the effects of a nanomaterial dielectric layer (here $\mathrm{TiO}_{2}$ material) deposited on the circuit and its impact in terms of electromagnetic propagation. As expected, it has been demonstrated that the deposit of proposed $\mathrm{TiO}_{2}$-material leads to a global decrease of the level of radiated EM fields with a relatively slight impact on $\mathrm{S}$ parameters.

Different prospects of this works are nowadays considered based upon the proposed methodology (i.e. microwave microscopy). In this framework, various nanomaterials may be used jointly with a multi-layers deposition procedure for ElectroMagnetic Compatibility / Integrity (EMC/EMI) purposes including 3D circuit structures. The consideration of the materials ageing should also be of interest. In that case, alternative EMC devices (e.g. Mode Stirred Reverberation Chambers, MSRCs) should be of great interest to complement use of microwave microscopy to characterize EMC implications due to use of nanomaterials.

\section{Acknowledgement}

This work was partially supported by French Burgundy Council through Innovation Program called Plan régional d'innovation (in French).

\section{References}

[1] A. Geetha, K.K.S. Kumar, C.R.K. Rao, M. Vijayan, D.C. Trivedi, "EMI shielding: Methods and materials - A review," Journal of Applied Polymer Science, vol. 112, 2073-2086, 2009.

[2] M.T. Ma, M. Kanda, M.L. Crawford, E.B. Larsen, "A review of electromagnetic compatibility/interference measurement methodologies," Proc. of the IEEE, Vol. 73, No. 3, March 1985.

[3] Y. Liu, B. Ravelo, J. Ben Hadj Slama, "Calculations of near-field emissions in frequency-domain into timedependent data with arbitrary wave form transient perturbations," Advanced electromagnetics Journal, Vol. 1, No. 2, August 2012.

[4] T. Hiraoka, et al. "Electric field distributions in microwave planar circuits by small coaxial probe and comparison with FDTD method.” 2005 European Microwave Conference. (2) IEEE, 2005.

[5] N.V. Kantartzis, T.D. Tsiboukis, "Modern EMC Analysis Techniques Volume I:Time-Domain Computational Schemes,"' Ed. Morgan \& Claypool, 2008. 
[6] N.V. Kantartzis, T.D. Tsiboukis, "Modern EMC Analysis Techniques Volume II: Models and Applications,"” Ed. Morgan \& Claypool, 2008.

[7] A. Désiré, A. Kriga, M. Youssouf, A. Siblini, J-P. Chatelon, M-F. Blanc-Mignon, B. Payet-Gervy, A. Piot, D. Dufeu, J-J. Rousseau, "Fabrication and characterization of micro-inductors deposited on magnetic thin and thick layers," Advanced electromagnetics Journal, Vol. 2, No. 3, December 2013.

[8] C.R. Paul, "Introduction to Electromagnetic Compatibility," John Wiley \& Sons Inc., Second edition, New York, 2006.

[9] P. Besnier, B. Démoulin, "Electromagnetic Reverberation Chambers,"' Ed. Wiley-ISTE, 2011.

[10] X. Dong, S. Deng, T. Hubing, D. Beetner, "Analysis of chip-level EMI using near-field magnetic scanning," 2004 Int. Symp. on EMC, Vol. 1, pp. 174-177, August 2004.

[11] J. Fan, "Near-Field Scanning for EM Emission Characterization", IEEE Electromagnetic Compatibility Magazine, Vol. 4(3), 2015.

[12] S.O. Land, O. Tereshchenko, M. Ramdani, F. Leferink, R. Perdirau, "Printed Circuit Board Permittivity Measurement Using Waveguide and Resonator Rings," Proceedings of EMC'14, Tokyo, Japan, 777780, 2014.

[13] D.S. Dixon, J. Masi, "Thin Coatings Can Provide Significant Shielding Against Low Frequency EMF Magnetic Fields," Proceedings of IEEE International Symposium on EMC, Denver, USA, 1035-1040, Aug. 1998.

[14] J. Rossignol, C. Plassard, E. Bourilot, O. Calonne, M. Foucault, E. Lesnewska, "Non-destructive technique to detect local buried defects in metal sample by scanning microwave microscope," Physical Review B, 83, 121409(R), 2011.

[15] G. Yingjie, I. Wolff. "A simple electric near field probe for microwave circuit diagnostics," IEEE MTTS International. (3). IEEE, 1996.

[16] C. Valdman, M.H.C. Dias, and J.C.A. dos Santos, J. C. A. . "Design and calibration of electric field probe to the frequency range of 2 to $3 \mathrm{GHz}$." IEEE Latin America Transactions, 6(7), pp. 557-564, 2008.

[17] Y. Wu, F. J. Rosenbaum. “ Mode chart for microstrip ring resonators (Short Papers)." IEEE Transactions on Microwave Theory and Techniques (21.7), pp. 487489, 1973.

[18] T. Ohno, K. Sarukawa, K. Tokieda, M. Matsumura, "Morphology of a $\mathrm{TiO} 2$ photocatalyst (Degussa, P-25) consisting of anatase and rutile crystalline phases," Journal of Catalysis, 203(1), pp. 82-86, 2001.
[19] O. Bunsho, et al. "What is Degussa (Evonik) P25? Crystalline composition analysis, reconstruction from isolated pure particles and photocatalytic activity test." Journal of Photochemistry and Photobiology A: Chemistry 216(2 ), pp. 179-182, 2010.

[20] U. Diebold, "The surface science of titanium dioxyde," Surface Science Report, 48(5), 53-229, 2003.

[21] G. Bailly, A. Harrabi, J. Rossignol, et al.,"Microwave gas sensing with a Microstrip InterDigital Capacitor: detection of NH 3 with $\mathrm{TiO}_{2}$ nanoparticles," Sensors and Actuators B: Chemical, 2016.

[22] G. Bailly, J. Rossignol, B. De Fonseca, et al, "Microwave gas sensing with hematite: shape effect on ammonia detection using pseudocubic, rhombohedral and spindle-like particles," ACS Sensors, 2016. 